\title{
Consensus of Multiple Second-Order Agents without Velocity Measurements
}

\author{
Yanping Gao, Long Wang, and Yingmin Jia
}

\begin{abstract}
This paper studies consensus problems of multiple second-order agents with time-varying topology. It is assumed that each possible interaction topology is strongly connected and balanced and each agent can only obtain the measurements of its position relative to its neighbors. A feasible protocol is proposed, and by applying the linear matrix inequality technique and the common Lyapunov function approach, some sufficient conditions for consensus are established. Simulations are provided to illustrate the effectiveness of the theoretical results.
\end{abstract}

\section{INTRODUCTION}

In many practical situations, all agents are required to reach an agreement on certain quantities of interest, which may be the formation center in formation control, the expected attitude in attitude alignment, and the destination in rendezvous problem. The estimations of the interest are often represented by agents' states. Such a problem is called consensus problem, which is one of fundamental research topics in the field of distributed control of multiple agents.

Consensus problems have received considerable attention recently. In [1], a discrete-time model of $n$ agents was proposed, where each agent's heading is updated based on the average of its own heading and its neighbors' headings. By simulation, it was shown that all the agents tend to move in the same heading although the set of each agent's neighbors is time-varying. A theoretical explanation for the above phenomenon was provided in [2] by introducing an undirected graph to model the interaction topology among agents. In [3], a directed graph was introduced and consensus problems for three cases, i.e., directed networks with fixed topology, directed networks with switching topology, and undirected networks with time-invariant communication delays, were investigated, respectively. In [4], an improved sufficient condition was obtained for consensus in directed networks with switching topology. Note that all the aforementioned work is concerned with agents with first-order or singleintegrator dynamics. In [5] and [6], two kinds of consensus problems of multiple second-order agents were discussed, respectively. In addition, with the fast development of the research on consensus problems, many new contents, such as finite-time consensus [10], agreement on random networks

This work was supported by NSFC (60674050, 60736022, and 60528007), National 863 Program (2006AA04Z258), and 11-5 project (A2120061303)

Y. Gao and L. Wang are with the Center for Systems and Control, College of Engineering and Key Laboratory of Machine Perception(Ministry of Education), Peking University, Beijing, 100871, P. R. China. gaoyanping@pku.edu.cn, longwang@pku.edu.cn

Y. Jia is with the Seventh Research Division, Beihang University, Beijing, 100083, P. R. China. ymjia@buaa.edu. cn
[11], consensus filter [12], and asynchronous consensus [13], have emerged. Interested readers can refer to the survey paper [14] and the references therein.

In the study of consensus problems, the design of protocols/algorithms is important. A protocol specifies that how each agent uses the information obtained by it to coordinate its behavior so that the states of all agents can reach a common value eventually. In most of the literature on consensus problems, such as [5] and [6], it is assumed that each agent can obtain the measurement of its full state. However, in practical situations, some information is unmeasurable because of technology limitations or environment disturbances. For example, in some cases, agents can not obtain any velocity information, and thus, the protocols proposed in [5] and [6] can not be implemented. Hence, it is realistic and favorable to consider the problem of design protocols in the case when each agent can only obtain the measurement of its partial state. However, there is only a little literature on this problem, such as [15], [16], [17]. In [16], tracking control of multiple agents with a leader was considered, while the leader's velocity is unmeasurable. In [17], consensus problems of multiple second-order agents were investigated in undirected networks with fixed topology, where each agent can only measure its positions relative to its neighbors. Note that all information is transmitted in real time in [15], [16], and [17]. Because of the physical properties of transmission medium and the capability of transmission bandwidth of networks, some information may not be transmitted among agents in real time, which results in the emergence of delays. Based on the above considerations, we investigate consensus problems of multiple second-order agents, where agents can only obtain the measurements of relative positions and the measurements may be with timedelays. Although our work is partly motivated by that in [17], they are different in the following three aspects. First, directed networks with switching topology are considered in our work, while just undirected networks with fixed topology are considered in [17]. Second, measurement delays, which is not studied in [17], is further investigated in our work. Third, our protocol is not a special case of that in [17].

An outline of this paper is shown as follows. In Section 2 , we present some concepts in graph theory and formulate the model to be studied. In Section 3, we derive the main results. In Section 4, simulations are provided to illustrate the effectiveness of the theoretical results. Finally, conclusion remarks are made in Section 5.

Throughout this paper, $I_{n} \in \mathbb{R}^{n \times n}$ is an identity matrix and $\mathbf{1}_{n}=\left[\begin{array}{llll}1 & 1 & \cdots & 1\end{array}\right]^{T} \in \mathbb{R}^{n} ; \otimes$ denotes Kronecker product; 
for symmetric matrix $A, A>0$ (resp., $A<0$ ) means $A$ is a positive definite (resp., negative definite) matrix; $\lambda_{\min }(B)$ represents the minimum eigenvalue of $B$.

\section{PRELIMINARIES}

\section{A. Graph theory}

Graph plays a key role in modeling the interaction topology among agents. We first introduce some basic definitions in graph [18].

A directed graph $\mathcal{G}$ consists of a vertex set $\mathcal{V}(\mathcal{G})$ and an edge set $\mathcal{E}(\mathcal{G})$, where $V(\mathcal{G})=\left\{\mathrm{v}_{1}, \cdots, \mathrm{v}_{n}\right\}$ and $\mathcal{E}(\mathcal{G}) \subset$ $\left\{\left(\mathrm{v}_{j}, \mathrm{v}_{i}\right): \mathrm{v}_{j}, \mathrm{v}_{i} \in \mathcal{V}(\mathcal{G})\right\}$. For edge $\left(\mathrm{v}_{j}, \mathrm{v}_{i}\right), \mathrm{v}_{j}$ is called the parent vertex of $\mathrm{v}_{i}$ and $\mathrm{v}_{i}$ is called the child vertex of $\mathrm{v}_{j}$. If two ends of an edge is the same vertex, then such an edge is called loop. The set of neighbors of vertex $\mathrm{v}_{i}$ is defined by $N\left(\mathcal{G}, \mathrm{v}_{i}\right)=\left\{\mathrm{v}_{j}:\left(\mathrm{v}_{j}, \mathrm{v}_{i}\right) \in \mathcal{E}(\mathcal{G})\right.$ and $j \neq$ $i\}$, and the associated index set is denoted by $N(\mathcal{G}, i)=$ $\left\{j: \mathrm{v}_{j} \in N\left(\mathcal{G}, \mathrm{v}_{i}\right)\right\}$. A (directed) path from $\mathrm{v}_{i_{1}}$ to $\mathrm{v}_{i_{k}}$ is a sequence, $\mathrm{v}_{i_{1}}, \cdots, \mathrm{v}_{i_{k}}$, of distinct vertices such that $\left(\mathrm{v}_{i_{j}}, \mathrm{v}_{i_{j+1}}\right) \in \mathcal{E}(\mathcal{G})$ for any $j=1, \cdots, k-1$. A directed graph $\mathcal{G}$ is strongly connected if there is a path between each pair of distinct vertices. A directed tree is a directed graph, where every vertex has exactly one parent except for one vertex, called root vertex, which has no parent and can be connected to any other vertices via pathes. A subgraph $\mathcal{G}_{s}$ of $\mathcal{G}$ is a graph such that $\mathcal{V}\left(\mathcal{G}_{s}\right) \subset \mathcal{V}(\mathcal{G})$ and $\mathcal{E}\left(\mathcal{G}_{s}\right) \subset \mathcal{E}(\mathcal{G})$. $\mathcal{G}_{s}$ is said to be a spanning subgraph if $\mathcal{V}\left(\mathcal{G}_{s}\right)=\mathcal{V}(\mathcal{G})$. For any $\mathrm{v}_{i}, \mathrm{v}_{j} \in \mathcal{V}\left(\mathcal{G}_{s}\right)$, if $\left(\mathrm{v}_{i}, \mathrm{v}_{j}\right) \in \mathcal{E}\left(\mathcal{G}_{s}\right) \Leftrightarrow\left(\mathrm{v}_{i}, \mathrm{v}_{j}\right) \in \mathcal{E}(\mathcal{G})$, then $\mathcal{G}_{s}$ is said to be an induced subgraph of $\mathcal{G}$, and we also say $\mathcal{G}_{s}$ is induced by $\mathcal{V}\left(\mathcal{G}_{s}\right)$. A spanning tree of $\mathcal{G}$ is a directed tree which is a spanning subgraph of $\mathcal{G}$. $\mathcal{G}$ is said to have a spanning tree if some edges of $\mathcal{G}$ form a spanning tree of $\mathcal{G}$.

A matrix is called nonnegative if each of its elements is nonnegative. A weighted directed graph $\mathcal{G}(A)$ is a directed graph $\mathcal{G}$ plus a nonnegative matrix $A=\left[a_{i j}\right] \in \mathbb{R}^{n \times n}$, where $a_{i j}>0 \Leftrightarrow\left(\mathrm{v}_{j}, \mathrm{v}_{i}\right) \in \mathcal{E}(\mathcal{G})$, and $a_{i j}$ is called the weight of edge $\left(\mathrm{v}_{j}, \mathrm{v}_{i}\right) . \mathcal{G}(A)$ is called balanced if $\sum_{j=1}^{n} a_{i j}=\sum_{j=1}^{n} a_{j i}, i=1, \cdots, n$. In this paper, we do not consider graphs with loops, namely, $a_{i i}=0, i=1, \cdots, n$. The Laplacian matrix $L=\left[l_{i j}\right] \in \mathbb{R}^{n \times n}$ of $\mathcal{G}(A)$ is defined as

$$
l_{i j}=\left\{\begin{array}{c}
-a_{i j}, i \neq j \\
\sum_{k=1}^{n} a_{i k}, i=j
\end{array}\right.
$$

and it has some properties as follows.

Lemma 1: ([3], [4]) Consider directed graph $\mathcal{G}(A)$.

(i) Zero is an eigenvalue of $L$, and $\mathbf{1}_{n}$ is the associated right eigenvector. Moreover, $\mathbf{1}_{n}$ is also the associated left eigenvector if $\mathcal{G}(A)$ is balanced.

(ii) Zero is an algebraically simple eigenvalue of $L$ and all the other eigenvalues are with positive real parts if and only if $\mathcal{G}(A)$ has a spanning tree.

\section{B. Protocol}

Consider $n$ agents with the following dynamics:

$$
\dot{x}_{i}(t)=v_{i}(t), \dot{v}_{i}(t)=u_{i}(t), i=1, \cdots, n,
$$

where $x_{i} \in \mathbb{R}^{m}$ and $v_{i} \in \mathbb{R}^{m}$ is the position and velocity vectors of agent $i$, respectively, and $u_{i}$ is the control input, called the protocol, to be designed based on the information obtained by agent $i$. Note that $x_{i}$ and $v_{i}$ can also represent other physical quantities. To facilitate the following analysis, we will consider the one-dimensional case, i.e., $m=1$. However, similar analysis can also be done for the higher dimensional case by means of Kronecker product.

Given $u_{i}, i=1, \cdots, n, u_{i}$ or multi-agent system (1) solves a consensus problem asymptotically if for any initial states, $\lim _{t \rightarrow \infty}\left(x_{r}(t)-x_{s}(t)\right)=0$ and $\lim _{t \rightarrow \infty}\left(v_{r}(t)-v_{s}(t)\right)=$ $0, r, s=1, \cdots, n$. Such a consensus problem can find application in formation control of multiple vehicles/robots (see, e.g., [19]).

In most of the literature on consensus problems of multiagent system (1), the protocols are implemented based on measurements of relative positions and velocities. For example, the protocol presented in [6] is

$$
\begin{aligned}
u_{i}(t)= & -\sum_{j \in N(\mathcal{G}, i)} a_{i j}\left(x_{i}(t)-x_{j}(t)\right) \\
& -k \sum_{j \in N(\mathcal{G}, i)} a_{i j}\left(v_{i}(t)-v_{j}(t)\right), k>0 .
\end{aligned}
$$

Hence, in the case that agents can only obtain measurements of relative positions, protocol (2) is infeasible. Here we will solve this problem and provide a feasible protocol.

In this paper, it is assumed that the interaction topology is time-varying and $t_{i+1}-t_{i} \geq \tau^{*}>0, i=0,1, \cdots$, where $t_{0}(=0), t_{1}, \cdots$ denote the times when the interaction topology changes. It is also assumed that the weight of each edge is chosen from a finite set $S_{1}$, which consists of finite positive numbers. Accordingly, the number of all possible interaction topologies among $n$ agents is finite. Let $S_{2}=\left\{\mathcal{G}\left(A_{1}\right), \cdots, \mathcal{G}\left(A_{N}\right)\right\}$ be a set of all possible topologies, where $A_{p}=\left[a_{i j}^{(p)}\right], p=1, \cdots, N$. For convenience, introduce a switching signal $\sigma:[0, \infty) \rightarrow \mathcal{P}$, where $\mathcal{P}=\{1, \cdots, N\}$. Let $L_{\sigma(t)}$ and $N_{i}(t)$ denote the Laplacian matrix of $\mathcal{G}\left(A_{\sigma(t)}\right)$ and the index set of neighbors of agent $i$ at time $t$, respectively. In addition, we make the following assumption,

(A1) $\mathcal{G}\left(A_{i}\right), i=1, \cdots, N$, are all strongly connected and balanced.

By the above description, the dynamics of agent $i$ is rewritten as

$$
\begin{aligned}
& \dot{x}_{i}(t)=v_{i}(t), \dot{v}_{i}(t)=u_{i}(t), \\
& y_{i j}(t)=x_{i}\left(t-\tau_{i j}(t)\right)-x_{j}\left(t-\tau_{i j}(t)\right), j \in N_{i}(t), \\
& i=1, \cdots, n,
\end{aligned}
$$

where $\tau_{i j}(t) \geq 0$. Let $y_{i}(t)=\sum_{j \in N_{i}(t)} a_{i j}^{(\sigma(t))} y_{i j}(t)$. Partly motivated by the theory of dynamic output feedback control, 
we provide the following protocol:

$$
\begin{aligned}
& \dot{z}_{i}(t)=a z_{i}(t)+b y_{i}(t)+c u_{i}(t), \\
& w_{i}(t)=d z_{i}(t)+f y_{i}(t), \\
& u_{i}(t)=-y_{i}(t)-k w_{i}(t)
\end{aligned}
$$

where parameters $a, b, c, d, f, k$ satisfy

$$
f c d>0,(b d-a f) c d>0, f k>0,
$$

which implies that $f, c d, b d-a f$, and $k$ are with the same sign.

Obviously, protocol (4) is implemented based on only the measurements of relative positions. In the following section, by applying the common Lyapunov function approach and the linear matrix inequality technique, we will present some sufficient conditions for protocol (4) to solve a consensus problem under arbitrary switching signals.

\section{MAIN RESULTS}

\section{A. Networks without time-delays}

We first consider the case without measurement delays, i.e., $\tau_{i j}(t)=0$. To facilitate the following analysis, let $\theta(t)=$ $\left[\begin{array}{llll}\theta_{1}^{T}(t & \theta_{2}^{T}(t) & \cdots & \theta_{n}^{T}(t)\end{array}\right]^{T}, \theta_{i}(t)=\left[\begin{array}{lll}x_{i}(t) & v_{i}(t) & w_{i}(t)\end{array}\right]^{T}, i=$ $1, \cdots, n$, then

$$
\dot{\theta}(t)=\left(I_{n} \otimes B_{1}-L_{\sigma} \otimes B_{2}\right) \theta(t)
$$

where

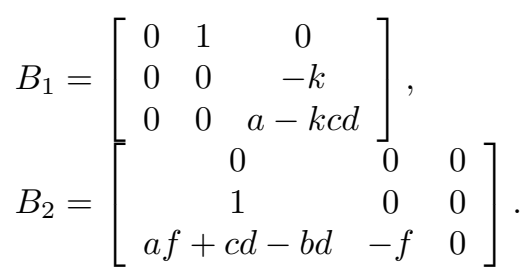

In order to apply the Lyapunov function approach, we make a state transformation for $\theta$. Let $U=\left[\frac{1}{\sqrt{n}} \mathbf{1}_{n} U_{1}\right]$ be an orthogonal matrix, then $U_{1}$ is full column rank, and under (A1), $U=\operatorname{diag}\left\{0, H_{\sigma}\right\}$, where $H_{\sigma} \in \mathbb{R}^{(n-1) \times(n-1)}$. Let $\delta(t)=\left(U^{-1} \otimes I_{3}\right) \theta(t)$, where $\delta(t)=\left[\delta_{1}^{T}(t) \widehat{\delta}^{T}(t)\right]^{T}, \widehat{\delta}(t)=$ $\left[\begin{array}{llll}\delta_{2}^{T}(t) & \cdots & \delta_{n}^{T}(t)\end{array}\right]^{T}, \quad \delta_{i}(t)=\left[\begin{array}{lll}\delta_{i 1}(t) & \delta_{i 2}(t) & \delta_{i 3}(t)\end{array}\right]^{T}, i=$ $1, \cdots, n$, then

$$
\begin{gathered}
\dot{\delta}_{1}(t)=B_{1} \delta_{1}(t) \\
\dot{\widehat{\delta}}(t)=\left(I_{n-1} \otimes B_{1}-H_{\sigma} \otimes B_{2}\right) \widehat{\delta}(t) .
\end{gathered}
$$

Obviously,

$$
\delta_{1}(t)=\frac{1}{\sqrt{n}} \sum_{i=1}^{n} \theta_{i}(t), \theta(t)=\frac{1}{\sqrt{n}} \mathbf{1}_{n} \otimes \delta_{1}(t)+\left(U_{1} \otimes I_{3}\right) \widehat{\delta}(t) .
$$

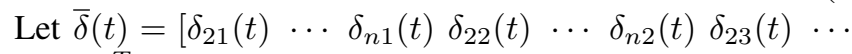
$\left.\delta_{n 3}(t)\right]^{T}$. Then

$$
\dot{\bar{\delta}}(t)=\Phi_{\sigma} \bar{\delta}(t)
$$

where

$$
\Phi_{\sigma}=\left[\begin{array}{ccc}
0 & I_{n-1} & 0 \\
-H_{\sigma} & 0 & -k I_{n-1} \\
(b d-a f-c d) H_{\sigma} & f H_{\sigma} & (a-k c d) I_{n-1}
\end{array}\right] .
$$

Remark 1: By (8), protocol (4) solves a consensus problem if system (9) is asymptotically stable. Hence, we focus on the stability of system (9).

To derive a main result, we need the following lemma.

Lemma 2: If $\mathcal{G}\left(A_{\sigma}\right)$ is strongly connected and balanced, then $H_{\sigma}+H_{\sigma}^{T}$ is positive definite.

Proof: Because $\mathcal{G}\left(A_{\sigma}\right)$ is strongly connected and balanced, $L_{\sigma}+L_{\sigma}{ }^{T}$ can be viewed as the Laplacian matrix of a connected graph. By Lemma 1 and $U^{-1}\left(L_{\sigma}+L_{\sigma}{ }^{T}\right) U=$ $\operatorname{diag}\left\{0, H_{\sigma}+H_{\sigma}^{T}\right\}$, all eigenvalues of $H_{\sigma}+H_{\sigma}^{T}$ are positive real numbers. Thus, $H_{\sigma}+H_{\sigma}^{T}$ is positive definite.

By applying the common Lyapunov function approach to study the stability of system (9), we can derive the following main result.

Theorem 1: Assume (A1) and (5) hold. If

$$
|f|>\max \left\{\left|b c d^{2}\right|,\left(1+\frac{1}{2 \beta}+|a c d|\right)^{2}\right\} \text {, }
$$

and

$$
|k|>\frac{2 a}{|c d|}-\frac{|b d-a f|}{2|f|} \gamma
$$

where

$$
\begin{aligned}
& \beta=\min _{\sigma=1, \cdots, N} \lambda_{\min }\left(\Gamma_{\sigma}\right), \Gamma_{\sigma}=H_{\sigma}+H_{\sigma}^{T}, \\
& \gamma=\min _{\sigma=1, \cdots, N} \lambda_{\min }\left(q_{\sigma}^{T} Q_{1 \sigma}^{-1} q_{\sigma}\right), \\
& Q_{1_{\sigma}}=\left[\begin{array}{cc}
-|b d-a f| \Gamma_{\sigma} & \sqrt{|f|} I_{n-1}-|f| \Gamma_{\sigma} \\
* & 2|c d| I_{n-1}-\frac{f|f|}{b d-a f} \Gamma_{\sigma}
\end{array}\right], \\
& q_{\sigma}=\left[\begin{array}{c}
-a \operatorname{sign}(f) I_{n-1}+|f|\left(\frac{2}{c d}-\frac{1}{b d-a f}\right) H_{\sigma}^{T} \\
-\left(\operatorname{sign}(f)+\frac{a|f|}{b d-a f}\right) I_{n-1}+\frac{2 f|f|}{(b d-a f) c d} H_{\sigma}^{T}
\end{array}\right],
\end{aligned}
$$

then protocol (4) solves a consensus problem under arbitrary switching signals. Furthermore, $\lim _{t \rightarrow \infty}\left[x_{i}(t)-\frac{1}{n} \sum_{i=1}^{n}\left(x_{i}^{*}+\right.\right.$ $\left.\left.t v_{i}^{*}\right)\right]=0, \lim _{t \rightarrow \infty} v_{i}(t)=\frac{1}{n} \sum_{i=1}^{n} v_{i}^{*}$, where $x_{i}^{*}=x_{i}(0)+$ $\frac{k}{(a-k c d)^{2}} \omega_{i}(0), v_{i}^{*}=v_{i}(0)+\frac{k}{a-k c d} \omega_{i}(0), i=1, \cdots, n$.

Proof: For system (9), consider the following Lyapunov function candidate: $V(t)=\bar{\delta}^{T}(t) P \bar{\delta}(t)$, where

$$
P=\left[\begin{array}{ccc}
\sqrt{|f|} & |c d| & -\operatorname{sign}(f) \\
* & \frac{c d|f|}{b d-a f} & \frac{-|f|}{b d-a f} \\
* & * & \frac{2|f|}{(b d-a f) c d}
\end{array}\right] \otimes I_{n-1} .
$$

By (10), $P$ is positive definite, which will be explained later. By calculation,

$$
\dot{V}(t)=\bar{\delta}^{T}(t)\left(P \Phi_{\sigma}+\Phi_{\sigma}^{T} P\right) \bar{\delta}(t)=\bar{\delta}^{T}(t) Q_{\sigma} \bar{\delta}(t),
$$

where

$$
Q_{\sigma}=\left[\begin{array}{cc}
Q_{1 \sigma} & q_{\sigma} \\
* & \frac{2|f|(2 a-k c d)}{(b d-a f) c d} I_{n-1}
\end{array}\right]
$$

By Schur complement (see [20]), $Q_{\sigma}<0$ if and only if $Q_{1 \sigma}<0$ and

$$
\frac{2|f|(2 a-k c d)}{(b d-a f) c d} I_{n-1}-q_{\sigma}^{T} Q_{1 \sigma}^{-1} q_{\sigma}<0 .
$$

By Lemma 2, $-|b d-a f| \Gamma_{\sigma}<0$. Thus, $Q_{1 \sigma}<0$ if and only if $\frac{1}{|b d-a f|}\left(\sqrt{|f|} I_{n-1}-|f| \Gamma_{\sigma}\right) \Gamma_{\sigma}^{-1}\left(\sqrt{|f|} I_{n-1}-\mid\right.$ 
$\left.f \mid \Gamma_{\sigma}\right)+2|c d| I_{n-1}-\frac{f|f|}{b d-a f} \Gamma_{\sigma}<0$, which is equivalent to

$$
2|c d| I_{n-1}+\frac{|f|}{|b d-a f|} \Gamma_{\sigma}^{-1}-\frac{2|f| \sqrt{|f|}}{|b d-a f|} I_{n-1}<0 .
$$

Clearly,

$$
\begin{aligned}
& 2|c d| I_{n-1}+\frac{|f|}{|b d-a f|} \Gamma_{\sigma}^{-1}-\frac{2|f| \sqrt{|f|}}{|b d-a f|} I_{n-1} \\
\leq & \frac{2\left|b c d^{2}\right|+2|a c d||f|+\frac{1}{\lambda_{\min }\left(\Gamma_{\sigma}\right)}|f|-2|f| \sqrt{|f|}}{|b d-a f|} I_{n-1} \\
= & -\frac{|f|\left(2 \sqrt{|f|}-\frac{1}{\lambda_{\min \left(\Gamma_{\sigma}\right)}}-2|a c d|\right)-2\left|b c d^{2}\right|}{|b d-a f|} I_{n-1} .
\end{aligned}
$$

From (10), we have $2 \sqrt{|f|}-\frac{1}{\beta}-2 \mid$ acd $\mid>2$ and $|f|>\mid$ $b c d^{2} \mid$. Hence, $|f|\left(2 \sqrt{|f|}-\frac{1}{\beta}-2 \mid\right.$ acd $\left.\mid\right)-2\left|b c d^{2}\right|>0$, which means $|f|\left(2 \sqrt{|f|}-\frac{1}{\lambda_{\min }\left(\Gamma_{\sigma}\right)}-2 \mid\right.$ acd $\left.\mid\right)-2 \mid$ $b c d^{2} \mid>0$, and (13) holds. Then $Q_{1 \sigma}<0$ for any $\sigma$. From (5) and (11), it follows that (12) holds for any $\sigma$. Thus, $Q_{\sigma}<0$ for any $\sigma$, namely, system (9) is asymptotically stable under arbitrary switching signals. From (8), we have

$$
\lim _{t \rightarrow \infty}\left(\theta_{i}(t)-\frac{1}{\sqrt{n}} \delta_{1}(t)\right)=0, i=1, \cdots, n .
$$

Therefore, protocol (4) solves a consensus problem under arbitrary switching signals.

Now we prove the second part of Theorem 1. Let

$$
M=\left[\begin{array}{lll}
m_{r 1} & m_{r 2} & m_{r 3}
\end{array}\right]=\left[\begin{array}{ccc}
1 & 1 & 1 \\
0 & 1 & a-k c d \\
0 & 0 & -\frac{(a-k c d)^{2}}{k}
\end{array}\right]
$$

then

$$
\begin{aligned}
& M^{-1}=\left[\begin{array}{lll}
m_{l 1} & m_{l 2} & m_{l 3}
\end{array}\right]^{T}=\left[\begin{array}{ccc}
1 & -1 & \frac{k(k c d-a+1)}{(a-k c d)^{2}} \\
0 & 1 & \frac{k}{a-k c d} \\
0 & 0 & -\frac{k}{(a-k c d)^{2}}
\end{array}\right], \\
& M^{-1} B_{1} M=J=\left[\begin{array}{ccc}
0 & 1 & 0 \\
0 & 0 & 0 \\
0 & 0 & a-k c d
\end{array}\right] .
\end{aligned}
$$

In the case of $a \leq 0$, it is obvious that $a-k c d=a-|k c d|<$ 0 . In the case of $a>0$, from $Q_{\sigma}<0$, we have $2 a-k c d<0$, which implies $a-k c d<0$. By (6), $\delta_{1}(t)=e^{B_{1} t} \delta_{1}(0)=$ $M e^{J t} M^{-1} \delta_{1}(0)$, and $\lim _{t \rightarrow \infty}\left[\delta_{1}(t)-\left(m_{r 1} m_{l 1}^{T}+m_{r 2} m_{l 2}^{T}+\right.\right.$ $\left.\left.t m_{r 1} m_{l 2}^{T}\right) \delta_{1}(0)\right]=0$, where

$$
\begin{aligned}
& \delta_{1}(0)=\frac{1}{\sqrt{n}}\left[\sum_{i=1}^{n} x_{i}(0) \sum_{i=1}^{n} v_{i}(0) \sum_{i=1}^{n} \omega_{i}(0)\right]^{T}, \\
& m_{r 1} m_{l 1}^{T}+m_{r 2} m_{l 2}^{T}=\left[\begin{array}{ccc}
1 & 0 & \frac{k}{(a-k c d)^{2}} \\
0 & 1 & \frac{k}{a-k c d} \\
0 & 0 & 0
\end{array}\right], \\
& m_{r 1} m_{l 2}^{T}=\left[\begin{array}{ccc}
0 & 1 & \frac{k}{a-k c d} \\
0 & 0 & 0 \\
0 & 0 & 0
\end{array}\right] .
\end{aligned}
$$

From (14), we have

$$
\begin{aligned}
& \lim _{t \rightarrow \infty}\left(x_{i}(t)-\frac{1}{\sqrt{n}} \delta_{11}(t)\right) \\
= & \lim _{t \rightarrow \infty}\left\{x_{i}(t)-\frac{1}{n} \sum_{i=1}^{n}\left[x_{i}(0)+\frac{k}{(a-k c d)^{2}} \omega_{i}(0)\right.\right. \\
& \left.\left.+t\left(v_{i}(0)+\frac{k}{a-k c d} \omega_{i}(0)\right)\right]\right\} \\
= & 0
\end{aligned}
$$

and $\lim _{t \rightarrow \infty} v_{i}(t)=\frac{1}{n} \sum_{i=1}^{n}\left(v_{i}(0)+\frac{k}{a-k c d} \omega_{i}(0)\right)$.

Finally, we prove $P>0$. By (5), $\frac{2|f|}{(b d-a f) c d}>0$. Let

$$
P_{1}=\left[\begin{array}{cc}
\sqrt{|f|} & \mid c d \\
* & \frac{c d|f|}{b d-a f}
\end{array}\right], p=\left[\begin{array}{c}
-\operatorname{sign}(f) \\
\frac{-|f|}{b d-a f}
\end{array}\right] .
$$

Then $P>0$ if and only if

$$
P_{1}>0 \text { and } \frac{2|f|}{(b d-a f) c d}-p^{T} P_{1}^{-1} p>0 .
$$

By calculation, (15) is equivalent to $\frac{|f| \sqrt{|f|}}{|b d-a f|}-|c d|>0$, which is satisfied if (13) is satisfied. Hence, we can derive $P>0$ from (10).

Remark 2: Actually, there exist $a, b, c, d, f, k$ such that (5), (10), and (11) are all satisfied. For example, in the case of $a<0, c>0$, and $d>0$, the three conditions are all satisfied if $f$ and $k$ are sufficiently large. To seek $a, b, c, d, f, k$ satisfying (5), (10), and (11), we can assume some of them are known, and then calculate the others.

\section{B. Networks with time-delays}

In this subsection, we take measurement delays into account, and assume that the delays are time-invariant, i.e., $\tau_{i j}(t)=\tau_{i j}>0$.

Let $\left\{\tau_{i j}: i=1, \cdots, n, \forall j \in N_{i}(t)\right\}=\left\{d_{1}, \cdots, d_{l}\right\}$, where $l \leq n(n-1)$. Notations $U, \theta, \delta_{1}, \widehat{\delta}, \bar{\delta}$, and $\Phi_{\sigma}$ in the above subsection are still be used here. Then

$$
\dot{\theta}(t)=\left(I_{n} \otimes B_{1}\right) \theta(t)-\sum_{j=1}^{l}\left(L_{\sigma_{j}} \otimes B_{2}\right) \theta\left(t-d_{j}\right),
$$

where $L_{\sigma_{j}}=\left[l_{r s}^{\left(\sigma_{j}\right)}\right] \in \mathbb{R}^{n \times n}$ and

$$
l_{r s}^{\left(\sigma_{j}\right)}=\left\{\begin{array}{cl}
-a_{r s}^{(\sigma)}, & s \in N_{r}(t) \text { and } \tau_{r s}=d_{j} \\
0, & s \in N_{r}(t) \text { and } \tau_{r s} \neq d_{j} \\
0, & s \notin N_{r}(t) \text { and } s \neq r \\
-\sum_{k=1, k \neq r}^{n} l_{r k}^{\left(\sigma_{j}\right)}, & s=r
\end{array} .\right.
$$

Clearly, $L_{\sigma}=\sum_{j=1}^{l} L_{\sigma_{j}}, L_{\sigma_{j}} \mathbf{1}_{n}=0, j=1, \cdots, l$. Under (A1), we have

$$
U^{-1} L_{\sigma} U=\left[\begin{array}{cc}
0 & 0 \\
0 & H_{\sigma}
\end{array}\right], U^{-1} L_{\sigma_{j}} U=\left[\begin{array}{cc}
0 & h_{\sigma_{j}} \\
0 & H_{\sigma_{j}}
\end{array}\right] .
$$

Following the manipulation described in the above subsection, we have

$$
\begin{aligned}
& \dot{\delta_{1}}(t)=B_{1} \delta_{1}(t)-\sum_{j=1}^{l}\left(h_{\sigma_{j}} \otimes B_{2}\right) \widehat{\delta}\left(t-d_{j}\right), \\
& \dot{\widehat{\delta}}(t)=\left(I_{n-1} \otimes B_{1}\right) \widehat{\delta}(t)-\sum_{j=1}^{l}\left(H_{\sigma_{j}} \otimes B_{2}\right) \widehat{\delta}\left(t-d_{j}\right),
\end{aligned}
$$


and

$$
\dot{\bar{\delta}}(t)=\Phi_{\sigma} \bar{\delta}(t)+\sum_{j=1}^{l} \Phi_{\sigma_{j}} \eta_{j}(t),
$$

where $\eta_{j}(t)=\bar{\delta}(t)-\bar{\delta}\left(t-d_{j}\right)$ and

$$
\Phi_{\sigma_{j}}=\left[\begin{array}{ccc}
0 & 0 & 0 \\
H_{\sigma_{j}} & 0 & 0 \\
(a f+c d-b d) H_{\sigma_{j}} & -f H_{\sigma_{j}} & 0
\end{array}\right] .
$$

Similarly, we focus on the stability of system (16). By means of the linear matrix inequalities technique, we can obtain the following main result.

Theorem 2: Assume (A1) and (5) hold. If (10) and (11) are both satisfied and there exist positive definite matrices $S, Q_{j}, R_{j}, j=1, \cdots, l$, such that

$$
\left[\begin{array}{ccc}
Q_{11} & Q_{12} & Q_{13} \\
* & Q_{22} & Q_{23} \\
* & * & Q_{33}
\end{array}\right]<0, \forall \sigma \in\{1,2, \cdots, N\},
$$

where

$$
\begin{aligned}
& Q_{11}=S \Phi_{\sigma}+\Phi_{\sigma}^{T} S, Q_{12}=\left[S \Phi_{\sigma_{1}}+Q_{1} \cdots S \Phi_{\sigma_{l}}+Q_{l}\right] \text {, } \\
& Q_{13}=\left[\begin{array}{lll}
\Phi_{\sigma}^{T} R_{1} & \cdots & \Phi_{\sigma}^{T} R_{l}
\end{array}\right], \\
& Q_{22}=\operatorname{diag}\left\{-Q_{1}-\frac{1}{d_{1}} R_{1}, \cdots,-Q_{l}-\frac{1}{d_{l}} R_{l}\right\}, \\
& Q_{23}=\left[\begin{array}{ccc}
\Phi_{\sigma_{1}}^{T} R_{1} & \cdots & \Phi_{\sigma_{1}}^{T} R_{l} \\
\vdots & \ddots & \vdots \\
\Phi_{\sigma_{l}}^{T} R_{1} & \cdots & \Phi_{\sigma_{l}}^{T} R_{l}
\end{array}\right] \text {, } \\
& Q_{33}=\operatorname{diag}\left\{-\frac{1}{d_{1}} R_{1}, \cdots,-\frac{1}{d_{l}} R_{l}\right\},
\end{aligned}
$$

then protocol (4) solves a consensus problem under arbitrary switching signals.

Proof: Consider the following Lyapunov functional candidate for system (16):

$$
\begin{aligned}
V(t)= & \bar{\delta}(t)^{T} S \bar{\delta}(t)+\sum_{j=1}^{l} \int_{t-d_{j}}^{t} \bar{\delta}(z)^{T} Q_{j} \bar{\delta}(z) d z \\
& +\sum_{j=1}^{l} \int_{t-d_{j}}^{t}\left(z-t+d_{j}\right) \dot{\bar{\delta}}(z)^{T} R_{j} \dot{\bar{\delta}}(z) d z,
\end{aligned}
$$

where $S, Q_{j}$ and $R_{j}$ are positive definite matrices satisfying (17). By Lemma 4 in [21],

$$
\begin{aligned}
\dot{V}(t)= & 2 \bar{\delta}^{T}(t) S \dot{\bar{\delta}}(t)+\sum_{j=1}^{l}\left[\bar{\delta}^{T}(t) Q_{j} \bar{\delta}(t)\right. \\
& \left.-\bar{\delta}\left(t-d_{j}\right)^{T} Q_{j} \bar{\delta}\left(t-d_{j}\right)\right] \\
& +\sum_{j=1}^{l}\left[-\int_{t-d_{j}}^{t} \dot{\bar{\delta}}(z)^{T} R_{j} \dot{\bar{\delta}}(z) d z+d_{j} \dot{\bar{\delta}}^{T}(t) R_{j} \dot{\bar{\delta}}(t)\right] \\
\leq \quad & 2 \bar{\delta}^{T}(t) S\left[\Phi_{\sigma} \bar{\delta}(t)+\sum_{j=1}^{l} \Phi_{\sigma_{j}} \eta_{j}(t)\right] \\
& +\sum_{j=1}^{l}\left[\bar{\delta}^{T}(t) Q_{j} \bar{\delta}(t)\right. \\
& \left.-\left(\bar{\delta}(t)-\eta_{j}(t)\right)^{T} Q_{j}\left(\bar{\delta}(t)-\eta_{j}(t)\right)\right] \\
& -\sum_{j=1}^{l} \frac{1}{d_{j}} \eta_{j}^{T}(t) R_{j} \eta_{j}(t) \\
& +\sum_{j=1}^{l} d_{j}\left(\Phi_{\sigma} \bar{\delta}(t)+\sum_{j=1}^{l} \Phi_{\sigma_{j}} \eta_{j}(t)\right)^{T} \\
& \times R_{j}\left(\Phi_{\sigma} \bar{\delta}(t)+\sum_{j=1}^{l} \Phi_{\sigma_{j}} \eta_{j}(t)\right) \\
& \xi^{T}(t) Q \xi(t),
\end{aligned}
$$

where $\xi(t)=\left[\begin{array}{llll}\bar{\delta}^{T}(t) & \eta_{1}^{T}(t) \cdots & \eta_{l}^{T}(t)\end{array}\right]^{T}$ and

$$
\begin{gathered}
=\left[\begin{array}{cccc}
S \Phi_{\sigma}+\Phi_{\sigma}^{T} S & S \Phi_{\sigma_{1}}+Q_{1} & \ldots & S \Phi_{\sigma_{l}}+Q_{l} \\
* & -Q_{1}-\frac{1}{d_{1}} R_{1} & \ldots & 0 \\
\vdots & \vdots & \ddots & \vdots \\
* & * & \cdots & -Q_{l}-\frac{1}{d_{l}} R_{l}
\end{array}\right] \\
+\sum_{j=1}^{l} d_{j}\left[\begin{array}{c}
\Phi_{\sigma}^{T} \\
\Phi_{\sigma_{1}}^{T} \\
\vdots \\
\Phi_{\sigma_{l}}^{T}
\end{array}\right] R_{j}\left[\begin{array}{cccc}
\Phi_{\sigma} & \Phi_{\sigma_{1}} & \cdots & \Phi_{\sigma_{l}}
\end{array}\right] .
\end{gathered}
$$

By Schur complement, $Q<0, \forall \sigma \in\{1, \cdots, N\}$ if and only if (17) holds. Thus, system (16) is asymptotically stable under arbitrary switching signals. From (8), we conclude that protocol (4) solves a consensus problem under arbitrary switching signals.

Remark 3: By the proof of Theorem 1, if (5), (10), and (11) are all satisfied, then there exists $P>0$ such that $P \Phi_{\sigma}+$ $\Phi_{\sigma}^{T} P<0, \forall \sigma \in\{1, \cdots, N\}$. For $S=P$, there exist positive definite matrices $R_{j}, Q_{j}$ such that $Q<0, \forall \sigma \in\{1, \cdots, N\}$ if $d_{1}, \cdots, d_{l}$ are all sufficiently small. Hence, under the condition of Theorem 2, (17) is feasible if $d_{1}, \cdots, d_{l}$ are all sufficiently small.

Theorem 2 is applicable to the case that the exact values of measurement delays are known in advance. In the case that we only know the upper bound of measurement delays, Theorem 2 can not be applied directly, while we can obtain the following corollary from Theorem 2 . Let $\widehat{d}$ denote the upper bound of all measurement delays.

Corollary 1: Assume (A1), (5), (10), and (11) all holds. Protocol (4) solves a consensus problem under arbitrary switching signals if $\widehat{d}<d^{*}$, where

$$
\begin{aligned}
& d^{*}=\operatorname{maximum} d \\
& \text { s.t. } S>0, Q_{j}>0, R_{j}>0, j=1, \cdots, l, \text { and }(17)^{\prime},
\end{aligned}
$$

where $(17)^{\prime}$ is obtained by replacing $d_{j}, j=1, \cdots, l$, in (17) with $d$.

It should be mentioned that $d^{*}$ is dependent on parameters of protocol (4).

\section{Simulations}

Consider the interaction topologies shown in Fig. 1, where the weight of each edge is 1. Thus, each graph in Fig. 1 is strongly connected and balanced. To find parameters satisfying (5), (10), and (11), we assume some of parameters are known. For example, let $a=-1, b=1, c=0.5$, and $d=2$, then we obtain $f>7.6368$ and $k>36.6880$. Choose $f=8$ and $k=38$.

Example 1: (Networks without time-delays) By Theorem 1, protocol (4) solves a consensus problem under arbitrary switching signals. For example, for any given initial states, the state trajectories of agents are depicted in Fig. 2, where the interaction topology is randomly chosen from those shown in Fig.1. 

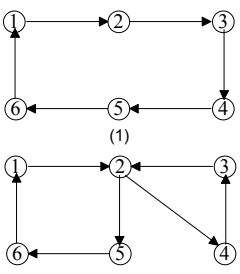

(3)

Fig. 1. Topologies.
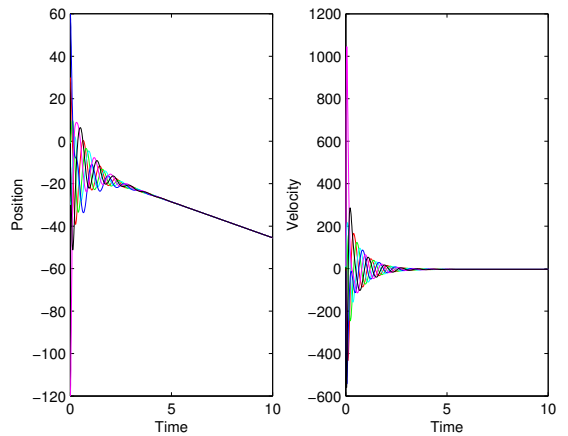

Fig. 2. Networks without time-delays.

Example 2: (Networks with time-delays) For convenience, assume all measurement delays are equal, i.e., $\tau_{i j}=$ $\tau$. In this case, $l=1$. By solving optimization problem (19), we obtain $d^{*}=0.0169$. Hence, for any $\tau<0.0169$, consensus can be reached under arbitrary switching signals by Theorem 2. For example, for any given initial states and $\tau=0.01$, the evolution of all agents' positions and velocities is shown in Fig.3, where the interaction topology is randomly chosen from those shown in Fig.1.

\section{CONCLUSION}

Consensus problems have been studied for multiple second-order agents with time-varying topology, where each agent can only obtain the measurements of its positions relative to its neighbors and each possible interaction topology is strongly connected and balanced. A feasible protocol with some parameters has been presented. Moreover, in the case without measurements delays, it has been shown that
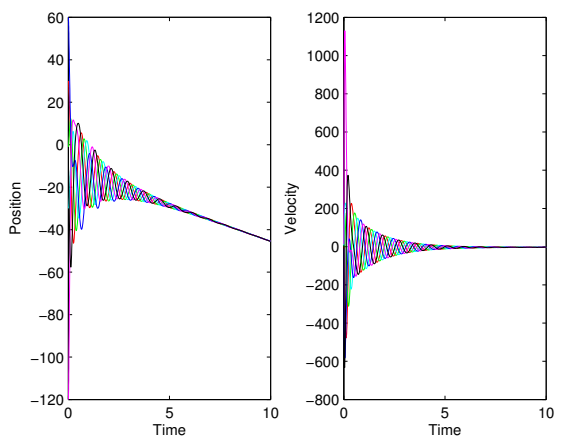

consensus can be reached if the parameters of the protocol satisfy some conditions; in the case with measurements delays, for the protocol with given parameters, it has been proved that consensus can be reached if the measurements delays are small enough, and an allowable upper bound of measurement delays has been obtained.

\section{REFERENCES}

[1] T. Vicsek, A. Czirók, E. Ben-Jacob, I. Cohen, and O. Schochet, Novel type of phase transition in a system of self-driven particles, Phys. Rev. Lett., vol. 75, no. 6, pp. 1226-1229, 1995.

[2] A. Jadbabaie, J. Lin, and A. S. Morse, Coordination of groups of mobile autonomous agents using nearest neighbor rules, IEEE Trans. Automat. Contr., vol. 48, no. 9, pp. 988-1001, 2003.

[3] R. Olfati-Saber and R. M. Murray, Consensus problems in networks of agents with switching topology and time-delays, IEEE Trans. Automat. Contr., vol. 49, no. 9, pp. 1520-1533, 2004.

[4] W. Ren and R. W. Beard, Consensus seeking in multiagent systems under dynamically changing interaction topologies, IEEE Trans. Automat. Contr., vol. 50, no. 5, pp. 655-661, 2005.

[5] G. Xie and L. Wang, Consensus control for a class of networks of dynamic agents: fixed topology, in: Proceedings of the IEEE Conference on Decision and Control, 2005, pp. 96-101.

[6] W. Ren and E. Atkins, Distributed multi-vehicle coordinated control via local information exchange, Int. J. Robust Nonlinear Control, vol. 17, no. 10-11, pp. 1002-1033, 2007.

[7] W. Wang and Jean-Jacques. E. Slotine, Contraction analysis of timedelayed communications and group cooperation, IEEE Trans. Automat. Contr., vol. 51, no. 4, pp. 712-717, 2006.

[8] L. Moreau, Stability of multiagent systems with time-dependent communication links, IEEE Trans. Automat. Contr., vol, 50, no. 2, pp. 169-182, 2005.

[9] L. Galbusera, M. P. E. Marciandi, P. Bolzern, and G. Ferrari-Trecate, Control schemes based on the wave equation for consensus in multiagent systems with double-integrator dynamics, in: Proceedings of the IEEE Conference on Decision and Control, 2007, pp. 1498-1503.

[10] F. Xiao, L. Wang, and Yingmin Jia, Fast information sharing in networks of autonomous agents, in: Proceedings of the American Control Conference, 2008, pp. 4388-4393.

[11] Y. Hatano and M. Mesbahi, Agreement over random networks, IEEE Trans. Autom. Contr., vol. 50, no. 11, pp. 1867-1872, 2005.

[12] W. Ren, R. W. Beard, and D. B. Kingston, Multi-agent Kalman consensus with relative uncertainty, in: Proceedings of the American Control Conference, 2005, pp. 1865-1870.

[13] V. Gazi, Stability of an asynchronous swarm with time-dependent communication links, IEEE Transactions on Systems, Man, and Cybernetics, Part B: Cybernetics, vol. 38, no. 1, 267-274, 2008.

[14] W. Ren, R. W. Beard, and E. M. Atkins, Information consensus in multi-vehicle cooperative control, IEEE Control Systems Magazine, vol. 27, no. 2, pp. 71-82, 2007.

[15] J. A. Fax and R. M. Murray, Information flow and cooperative control of vehicle formations, IEEE Trans. Automat. Contr., vol. 49, no. 9, pp. 1465-1476, 2004.

[16] Y. Hong, J. Hu, and L. Gao, Tracking control for multi-agent consensus with an active leader and variable topology, Automatica, vol. 42, no. 7, pp. 1177-1182, 2006.

[17] W. Ren, On consensus algorithms for double-integrator dynamics, in: Proceedings of the IEEE Conference on Decision and Control, 2007, pp. 2295-2300.

[18] C. Godsil and G. Royal, Algebraic graph theory, Springer- Verlag, New York, 2001.

[19] W. Ren, Consensus strategies for cooperative control of vehicle formations, IET Control Theory Appl., vol. 1, no. 2, pp. 505-512, 2007.

[20] B. Boyd, L.E. Ghaoui, E. Feron, and V. Balakrishnan, Linear Matrix Inequalities in System and Control Theory, SIAM, Philadelphia, PA, 1994.

[21] Y. Sun, L. Wang, and G. Xie, Average consensus in networks of dynamic agents with switching topologies and multiple time-varying delays, Systems \& Control Lett., vol. 57, no. 2, pp. 175-183, 2008.

Fig. 3. Networks with time-delays. 